\title{
Comparison of the atomic structure of InP amorphized by electronic or nuclear ion energy-loss processes
}

\author{
C. S. Schnohr, ${ }^{1}$ P. Kluth, ${ }^{1}$ A. P. Byrne, ${ }^{2,3}$ G. J. Foran, ${ }^{4}$ and M. C. Ridgway ${ }^{1}$ \\ ${ }^{1}$ Department of Electronic Materials Engineering, Research School of Physical Sciences and Engineering, \\ Australian National University, Canberra ACT 0200, Australia \\ ${ }^{2}$ Department of Physics, College of Science, Australian National University, Canberra ACT 0200, Australia \\ ${ }^{3}$ Department of Nuclear Physics, Research School of Physical Sciences and Engineering, Australian National University, \\ Canberra ACT 0200, Australia \\ ${ }^{4}$ Australian Nuclear Science and Technology Organisation, Menai NSW 2234, Australia
}

(Received 2 August 2007; published 20 February 2008)

\begin{abstract}
InP was amorphized by ion irradiation in two very different regimes: (i) $185 \mathrm{MeV} \mathrm{Au}$ irradiation, where the energy loss was predominantly via inelastic processes (electronic stopping), or (ii) Se irradiation in an energy range of $0.08-7 \mathrm{MeV}$, where elastic processes (nuclear stopping) were dominant. The structural parameters of the amorphous phase were determined for as-irradiated and thermally relaxed samples using extended x-ray absorption fine structure spectroscopy. Despite the fundamentally different energy transfer mechanisms, no significant difference in the atomic structure of the two amorphized samples was observed. We attribute this result to a common "melt and quench" process responsible for amorphization. In fact, the measured structural parameters for the amorphized samples, including the fraction of homopolar In-In bonding, were consistent with simulations of the amorphous phase produced by assuming a quench from the melt.
\end{abstract}

DOI: 10.1103/PhysRevB.77.073204

PACS number(s): 61.43.Dq, 61.05.cj, 61.80.Jh, 81.05.Ea

Amorphous semiconductors and their atomic-scale structure are of technological as well as of scientific interest. Ion irradiation induced amorphization of InP has been extensively studied in the keV energy range, ${ }^{1}$ and extended $\mathrm{x}$-ray absorption fine structure (EXAFS) spectroscopy has been successfully applied to determine the structural parameters of the amorphous phase. ${ }^{2-4}$ The amorphization of InP by swift heavy ions (SHIs) was first reported by Herre et al. ${ }^{5}$ The energy-loss processes for these two regimes are fundamentally different. Incoming ions from the $\mathrm{keV}$ to low $\mathrm{MeV}$ range deposit their energy predominantly via ballistic collisions with the target atoms (nuclear stopping), resulting in atomic displacements if sufficient energy is transferred. In contrast, incoming SHIs primarily interact with the target electrons (electronic stopping), leading to excitation and ionization of the electronic system. Given the differences in the energy deposition processes, one may well expect differences in the resulting amorphous phase structures. We now present a detailed comparison of the atomic-scale structural parameters obtained with EXAFS measurements of InP amorphized by either nuclear or electronic energy-loss processes. Despite fundamentally different energy transfer mechanisms, we find that the resulting amorphous phase structure is very similar for the two regimes.

For the EXAFS measurements, $\mathrm{InP} / \mathrm{In}_{0.53} \mathrm{Ga}_{0.47} \mathrm{As} / \mathrm{InP}$ heterostructures $[2.75 \mu \mathrm{m} / 50 \mathrm{~nm} /(100)$ substrate] were first grown by metal organic chemical vapor deposition. For amorphization induced by electronic interactions (SHI sample), the material was irradiated at room temperature with $185 \mathrm{MeV} \mathrm{Au}{ }^{13+}$ ions to a fluence of $3 \times 10^{13} \mathrm{~cm}^{-2}$. At this ion energy, the damage due to the electronic energy loss extends 5-10 $\mu \mathrm{m}$ into the sample, ${ }^{6,7}$ thus spanning the entire depth of the InP epilayer. The InP substrate and the $\mathrm{In}_{0.53} \mathrm{Ga}_{0.47} \mathrm{As}$ layer were then removed by selective chemical etching, first using the intermediate layer and then the epil- ayer as etch stops. For amorphization due to nuclear energy deposition, the same process of removing the InP substrate and the $\mathrm{In}_{0.53} \mathrm{Ga}_{0.47} \mathrm{As}$ layer was performed prior to irradiation. The InP films, bonded to $\mathrm{Si}$ for support, were then amorphized at liquid nitrogen temperature with multipleenergy Se irradiations. Ion energies ranged from $80 \mathrm{keV}$ to $7 \mathrm{MeV}$ with the fluences chosen to produce a constant value of total vacancy production over the extent of the InP epilayer (total fluence of $8.5 \times 10^{15} \mathrm{~cm}^{-2}$ ). ${ }^{8}$ Hereafter, this sample will be referred to as the low energy ion (LEI) sample. For irradiation in both energy regimes, the material was mounted on the sample holder with $\mathrm{C}$ paste to facilitate thermal contact and the areal power density was kept below $0.9 \mathrm{~W} \mathrm{~cm}^{-2}$. The amorphous nature of the material after irradiation under these conditions was confirmed by Rutherford backscattering spectrometry in a channeling configuration $(\mathrm{RBS} / \mathrm{c})$. A crystalline InP reference sample was also prepared from the heterostructures as above. All samples were finely crushed and mixed with $\mathrm{BN}$ prior to the EXAFS measurements. A detailed description of the preparation is given in Refs. 2 and 9.

EXAFS measurements of the In $K$ edge were performed in transmission mode at a temperature of $\sim 20 \mathrm{~K}$ using beamline NW10A at the Photon Factory, Japan. After the measurement, the SHI and LEI samples were annealed at $150{ }^{\circ} \mathrm{C}$ for $1 \mathrm{~h}$ and then remeasured. Such low temperature annealing leads to structural relaxation with the aim of achieving the intrinsic amorphous phase governed by the minimum-energy configuration. ${ }^{3,4}$ The data were processed and analyzed using the IFEFFIT $\operatorname{code}^{10}$ and the corresponding user interfaces ATHENA and ARTEMIS. ${ }^{11}$ Fitting was performed in radial space over a range of $R=1.5-2.9 \AA$ after Fourier transformation (FT) over a photoelectron wave number $(k)$ range of $k=2-14 \AA^{-1}$ with multiple $k$ weights $=2,3$, and 4 . Phase shifts and scattering amplitudes were calculated 

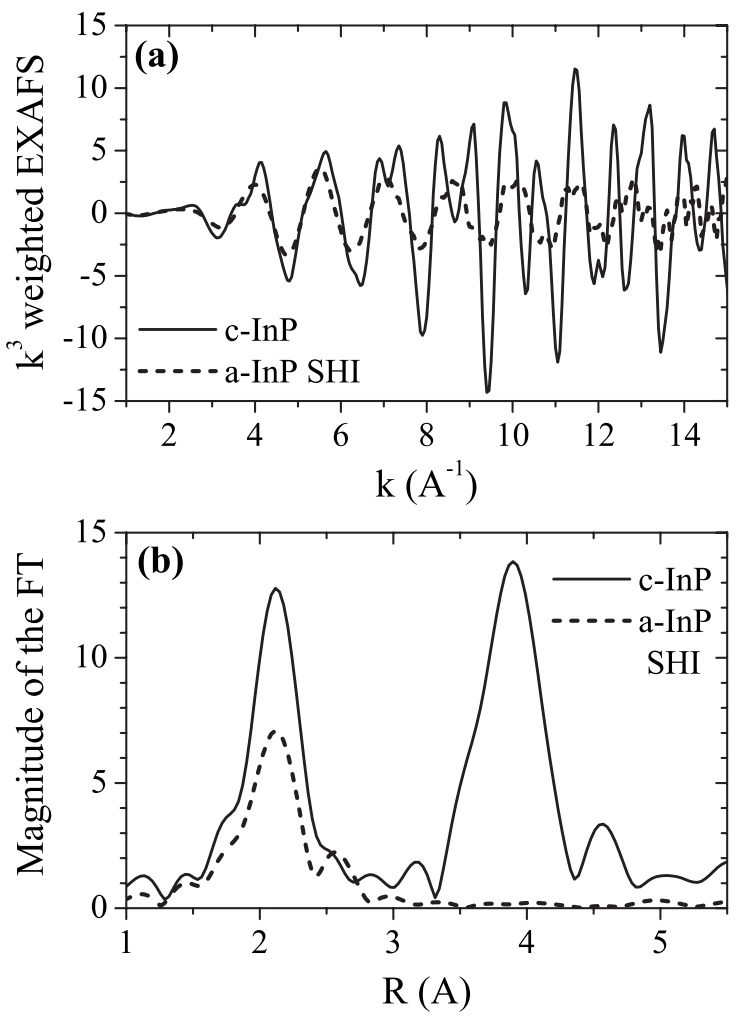

FIG. 1. (a) $k^{3}$-weighted EXAFS spectra of crystalline InP (c-InP, solid line) and amorphous InP as-irradiated by $185 \mathrm{MeV}$ $\mathrm{Au}^{13+}$ ( $a$-InP SHI, dashed line) versus the photoelectron wave number $k$. (b) Fourier transforms of the spectra shown in (a) as a function of the non-phase-corrected radial distance $R$ from the absorber.

$a b$ initio with FEFF8. ${ }^{12}$ The amplitude reduction factor $\left(S_{0}^{2}\right)$ and the threshold energy $\left(E_{0}\right)$ were determined from the crystalline standard and were fixed while fitting the amorphous samples.

Figure 1(a) shows the $k^{3}$-weighted EXAFS signal as a function of $k$ for the crystalline standard and the as-irradiated SHI sample. The EXAFS spectrum of the crystalline material shows a complex structure indicating the presence of several different frequencies. This is confirmed in the corresponding FT [Fig. 1(b)], which contains three distinct peaks at $R$ $\sim 2.1 \AA, R \sim 3.9 \AA$, and $R \sim 4.6 \AA$ representing scattering from the first $(\mathrm{P})$, second (In), and third $(\mathrm{P})$ nearest neighbors (NNs), respectively. In contrast, the EXAFS of the irradiated sample is dominated by a single frequency and only the first shell peak is observed in the FT. Structural disorder in amorphous materials is typically sufficient to prevent coherent scattering from beyond the first shell. The absence of higher shell peaks in the EXAFS spectrum is a characteristic feature of amorphous semiconductors and has been previously observed after ion irradiation in the LEI regime. ${ }^{4}$ Our EXAFS measurements are consistent with this behavior and confirm the formation of the amorphous phase after SHI irradiation as previously demonstrated by RBS/c, transmission electron microscopy, and x-ray diffraction. ${ }^{5,13}$ In addition to the reduction in amplitude of the first NN P peak, a small second peak at $R \sim 2.6 \AA$ is apparent in Fig. 1(b), resulting from the presence of a second component-In atoms-in the first shell of the amorphous material.

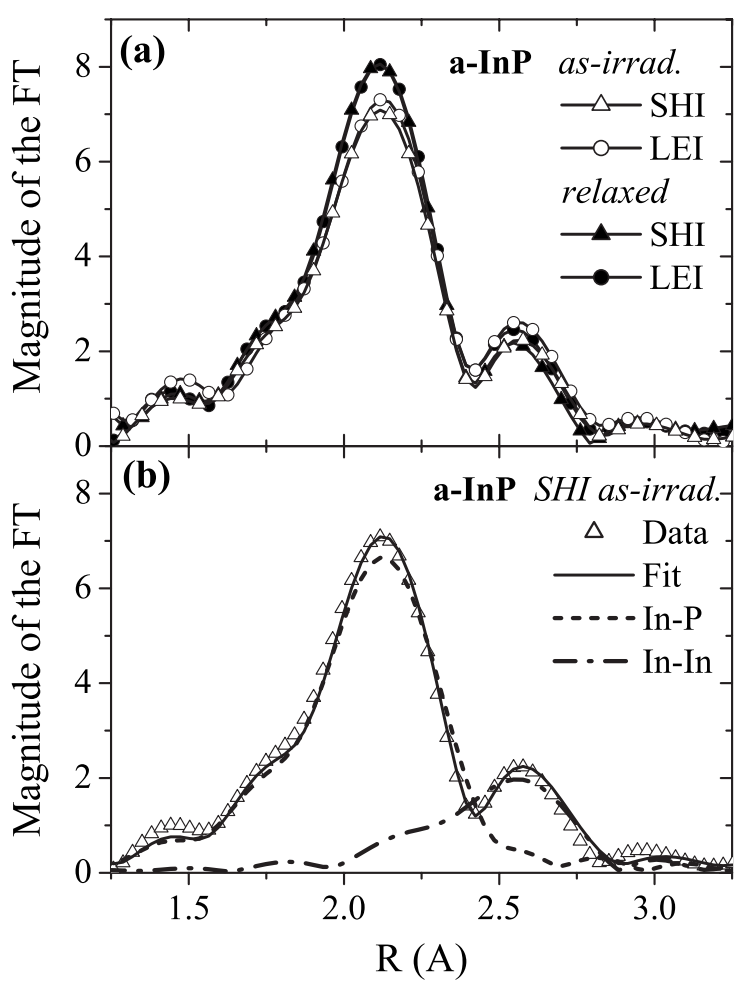

FIG. 2. (a) Fourier transforms of the experimental EXAFS of amorphous InP versus the non-phase-corrected radial distance $R$. Shown are the spectra of InP amorphized by $185 \mathrm{MeV} \mathrm{Au}^{13+}(a$-InP SHI, triangles) and by multiple-energy Se irradiation in the range of $0.08-7 \mathrm{MeV}$ ( $a$-InP LEI, circles) for the as-irradiated and relaxed samples (open and full symbols, respectively). For clarity, only every second data point is represented by a symbol. (b) Experimental data from (a) and the best fit as a function of $R$ for the as-irradiated SHI sample. Every data point is now represented by a symbol. Also shown are the two contributions arising from In-P and In-In bonds in the first shell (dotted and dash-dotted lines, respectively).

Figure 2(a) plots the FT versus $R$ for the SHI and LEI samples measured in as-irradiated and relaxed forms. Clearly, the spectra for the two samples amorphized in the different energy regimes are very similar both before and after relaxation. The relaxed samples are characterized by an increase in amplitude of the P peak, while the overall features of the amorphous phase are retained. All four spectra exhibit the smaller peak at $R \sim 2.6 \AA$ associated with In-In bonds. The contribution of these homopolar bonds is further shown in Fig. 2(b), plotting the FT of the as-irradiated SHI sample and the best fit including the individual scattering contributions of $\mathrm{P}$ and In atoms in the first shell. The structural parameters, including the coordination number $N$, bond length $r$, and Debye-Waller factor $\sigma^{2}$, are summarized in Table I for the different amorphous samples and the crystalline standard.

Comparing the as-irradiated and crystalline materials, amorphization yields a decrease in heteropolar coordination number and an increase in bond length and Debye-Waller factor. Combined with the approximately $15 \%$ In-In bonding fraction, these trends are very similar to the behavior previously observed after irradiation in the LEI regime., ${ }^{2,3}$ Relax- 
TABLE I. Structural parameters obtained for crystalline $\operatorname{InP}(c-\operatorname{InP})$ and $\operatorname{InP}$ amorphized by either $185 \mathrm{MeV}^{\mathrm{Au}}{ }^{13+}$ irradiation $(a-\operatorname{InP}$ SHI) or by multiple-energy Se irradiation in the range of 0.08-7 MeV ( $a$-InP LEI). The coordination number $(N)$, bond length $(r)$, and Debye-Waller factor $\left(\sigma^{2}\right)$ for the first shell In-P and In-In bonds are listed. Also given are the total coordination number $\left(N_{t o t}\right)$ and the percentage of In-In bonds $\left(\%_{\text {In-In }}\right)$.

\begin{tabular}{|c|c|c|c|c|c|c|c|c|c|}
\hline & & \multicolumn{3}{|c|}{ In-P } & \multicolumn{3}{|c|}{ In-In } & \multirow[b]{2}{*}{$N_{\text {tot }}$} & \multirow[b]{2}{*}{$\%_{\text {In-In }}$} \\
\hline & & $N$ & $r(\AA)$ & $\sigma^{2}\left(10^{-3} \AA^{2}\right)$ & $N$ & $r(\AA)$ & $\sigma^{2}\left(10^{-3} \AA^{2}\right)$ & & \\
\hline \multicolumn{2}{|c|}{$c-\operatorname{InP}$} & 4 fixed & $2.542 \pm 0.003$ & $2.3 \pm 0.2$ & & & & 4 & \\
\hline \multirow[t]{2}{*}{$a$-InP SHI } & As-irradiated & $3.43 \pm 0.26$ & $2.575 \pm 0.003$ & $5.5 \pm 0.8$ & $0.70 \pm 0.27$ & $2.783 \pm 0.009$ & $5.8 \pm 2.1$ & $4.13 \pm 0.53$ & 17 \\
\hline & Relaxed & $3.54 \pm 0.27$ & $2.569 \pm 0.004$ & $4.8 \pm 0.7$ & $0.66 \pm 0.15$ & $2.767 \pm 0.013$ & 5.8 fixed & $4.20 \pm 0.42$ & 16 \\
\hline \multirow[t]{2}{*}{$a$-InP LEI } & As-irradiated & $3.47 \pm 0.30$ & $2.576 \pm 0.004$ & $5.5 \pm 1.0$ & $0.63 \pm 0.27$ & $2.782 \pm 0.008$ & $4.4 \pm 2.2$ & $4.10 \pm 0.57$ & 15 \\
\hline & Relaxed & $3.57 \pm 0.23$ & $2.570 \pm 0.003$ & $5.0 \pm 0.6$ & $0.57 \pm 0.10$ & $2.771 \pm 0.008$ & 4.4 fixed & $4.14 \pm 0.33$ & 14 \\
\hline
\end{tabular}

ation leads to a small increase in heteropolar coordination number, while the Debye-Waller factor decreases slightly. Due to the strong correlation between these two parameters, the In-In Debye-Waller factor has been fixed to the asirradiated value while fitting the relaxed spectra following the analysis of Azevedo et al. $^{3}$ This yields a small reduction of the homopolar coordination number upon relaxation. The In-P and In-In bond lengths are smaller in the relaxed samples compared to as-irradiated values. In summary, relaxation yields a change of the structural parameters toward those of a crystalline material, again consistent with previous findings for samples amorphized in the LEI regime. ${ }^{3}$ The current work shows that a significant percentage of In-In bonds is present in the relaxed samples for both energy regimes and, thus, confirms that chemical disorder is a characteristic feature of amorphous InP and, most likely, the III-V semiconductors in general.

We note that the relaxation temperature is well below that required for recrystallization. Relaxation, therefore, yields a small change in the amorphous phase structure, reducing disorder but not inducing recrystallization. ${ }^{3}$ The ability to measure such subtle changes [see Fig. 2(a) and Table I] demonstrates the sensitivity of the EXAFS technique and shows that the atomistic processes operative during relaxation are the reduction of both structural and chemical disorders. EXAFS is, in fact, very well suited to the measurement of relative differences in amorphous phase structure. Previous examples include slight changes in the structural parameters of amorphous Ge resulting from (i) fabrication by sputtering or evaporation, ${ }^{14}$ (ii) formation by ion implantation as a function of ion dose, ${ }^{15}$ and (iii) low-temperature thermal annealing as a function of temperature. ${ }^{16} \mathrm{~A}$ recent extreme is the femtometer accuracy achieved with EXAFS for differential interatomic distance measurements of a polycrystalline FeCo alloy. $^{17}$

No significant difference is observed when comparing the In-P contribution of the SHI sample with that of the LEI sample. By comparing the In-In contributions, a small difference is apparent in the amplitude of the peak. Though this difference is stable under various fitting conditions, the magnitude of the experimental uncertainty inhibits us from an unambiguous assignment of its physical origin. We, therefore, conclude that amorphization in both energy regimes leads to a similar amorphous phase structure despite the fundamentally different energy transfer mechanisms.

Processes induced by ion irradiation are nonequilibrium in nature, and different models have been proposed to account for experimental findings. On one hand, an extended thermal spike model has recently been applied to predict the irradiation conditions necessary to produce continuous amorphous tracks in InP and good agreement with experimental data was obtained. ${ }^{7}$ The thermal spike model $^{18}$ assumes that electronic energy deposition above a certain threshold leads to melting of the material along the ion trajectory which, in the present case, is subsequently quenched into the amorphous phase during rapid resolidification. Our SHI irradiation conditions fall within the energy range where the calculated maximum temperature exceeds the melting point and a "melt and quench" process is expected. ${ }^{7}$ On the other hand, damage production in the LEI regime is usually associated with collision cascades and the formation, accumulation, and growth of various types of defects. ${ }^{19-22}$ Studies of InP irradiated under conditions very similar to our LEI irradiation demonstrate that a direct amorphization process is operative, where amorphous material is produced within a single ion impact. ${ }^{1,23}$ Nordlund et al. have investigated the amorphization mechanism during LEI irradiation using molecular dynamics (MD) simulations. For both Ge (Ref. 24) and GaAs, ${ }^{25}$ they report that recoils of several $\mathrm{keV}$ lead to molten regions in the material, which subsequently form amorphous pockets upon cooling. In contrast, collision cascades in $\mathrm{Si}$ are less dense and, hence, less damage with a higher fraction of isolated defects results. ${ }^{24}$ Following their discussion and supported by the direct amorphization observed experimentally, we expect a process similar to that in Ge and GaAs to take place in InP for our LEI irradiation conditions. Thus, independent of the energy transfer mechanism, amorphization in both regimes proceeds via a similar process. As a consequence, our SHI and LEI samples have a nearly identical atomic structure. Amorphization of InP by quenching from the liquid has been modeled with MD simulations by Lewis et $a l .{ }^{26}$ Homopolar bonds are present in the liquid phase and are retained ( $8 \%$ of all bonds) after quenching into the amorphous phase. The authors conclude that the presence of chemical disorder is necessary to stabilize the amorphous phase. Our SHI and LEI samples, in both the as-irradiated 
and relaxed states, contain a significant fraction of In-In bonds. Given the additional agreement of our experimental results with theoretical predictions of the amorphous phase structure resulting from a quench from the melt, our results could be viewed as indirect evidence that the amorphization process common to both energy regimes is, indeed, quenching of molten regions. These molten regions were formed due to the energy loss of the incoming ions, either via electronic stopping for the SHI case or nuclear stopping for the LEI case.

In conclusion, structural parameters of InP amorphized by ion irradiation where the energy loss was dominated by either electronic (SHI) or nuclear (LEI) stopping were determined from EXAFS measurements. No significant difference in the atomic structure of the two samples amorphized in the different energy regimes was apparent despite the fundamentally different energy transfer mechanisms. In agreement with the thermal spike model (SHI) and MD simulations for collision cascades (LEI), the common amorphous phase structure reported herein suggests that the energy deposition leads, in both cases, to a molten region that is subsequently quenched into the amorphous phase.

We thank H. H. Tan for the growth of the heterostructures. We also thank the staff at the ANU Heavy-Ion Facility and at the beamline NW10A of the Photon Factory for their continued support. The authors were supported by the Australian Synchrotron Research Program. P.K., A.P.B., and M.C.R. further acknowledge the support of the Australian Research Council.
${ }^{1}$ E. Wendler, T. Opfermann, and P. I. Gaiduk, J. Appl. Phys. 82, 5965 (1997).

${ }^{2}$ C. J. Glover, M. C. Ridgway, K. M. Yu, G. J. Foran, T. W. Lee, Y. Moon, and E. Yoon, Appl. Phys. Lett. 74, 1713 (1999).

${ }^{3}$ G. de M. Azevedo, C. J. Glover, M. C. Ridgway, K. M. Yu, and G. J. Foran, Phys. Rev. B 68, 115204 (2003).

${ }^{4}$ M. C. Ridgway, C. J. Glover, G. de M. Azevedo, S. M. Kluth, K. M. Yu, and G. J. Foran, Nucl. Instrum. Methods Phys. Res. B 238, 294 (2005).

${ }^{5}$ O. Herre, W. Wesch, E. Wendler, P. I. Gaiduk, F. F. Komarov, S. Klaumünzer, and P. Meier, Phys. Rev. B 58, 4832 (1998).

${ }^{6}$ W. Wesch, O. Herre, P. I. Gaiduk, E. Wendler, S. Klaumünzer, and P. Meier, Nucl. Instrum. Methods Phys. Res. B 146, 341 (1998).

${ }^{7}$ A. Kamarou, W. Wesch, E. Wendler, A. Undisz, and M. Rettenmayr, Phys. Rev. B 73, 184107 (2006).

${ }^{8} 4.0 \times 10^{15} \mathrm{~cm}^{-2}$ at $7.0 \mathrm{MeV}, 1.4 \times 10^{15} \mathrm{~cm}^{-2}$ at $3.5 \mathrm{MeV}, 0.8$ $\times 10^{15} \mathrm{~cm}^{-2}$ at $2.0 \mathrm{MeV}, 0.7 \times 10^{15} \mathrm{~cm}^{-2}$ at $1.0 \mathrm{MeV}, 0.8$ $\times 10^{15} \mathrm{~cm}^{-2}$ at $0.3 \mathrm{MeV}$, and $0.8 \times 10^{15} \mathrm{~cm}^{-2}$ at $0.08 \mathrm{MeV}$.

${ }^{9}$ C. S. Schnohr, P. Kluth, A. P. Byrne, G. J. Foran, and M. C. Ridgway, Nucl. Instrum. Methods Phys. Res. B 257, 293 (2007).

${ }^{10}$ M. Newville, J. Synchrotron Radiat. 8, 322 (2001).

${ }^{11}$ B. Ravel and M. Newville, J. Synchrotron Radiat. 12, 537 (2005).

${ }^{12}$ A. L. Ankudinov, B. Ravel, J. J. Rehr, and S. D. Conradson, Phys. Rev. B 58, 7565 (1998).

${ }^{13}$ N. Darowski, I. Zizak, and G. Schumacher, Physica B 357, 118
(2005).

${ }^{14}$ G. Dalba, P. Fornasini, M. Grazioli, and F. Rocca, Phys. Rev. B 52, 11034 (1995).

${ }^{15}$ M. C. Ridgway, C. J. Glover, K. M. Yu, G. J. Foran, C. Clerc, J. L. Hansen, and A. Nylandsted Larsen, Phys. Rev. B 61, 12586 (2000).

${ }^{16}$ C. J. Glover, M. C. Ridgway, K. M. Yu, G. J. Foran, D. DesnicaFrankovic, C. Clerc, J. L. Hansen, and A. Nylandsted-Larsen, Phys. Rev. B 63, 073204 (2001).

${ }^{17}$ R. F. Pettifer, O. Mathon, S. Pascarelli, M. D. Cooke, and M. R. J. Gibbs, Nature (London) 435, 78 (2005).

${ }^{18}$ M. Toulemonde, C. Dufour, and E. Paumier, Phys. Rev. B 46, 14362 (1992).

${ }^{19}$ J. F. Gibbons, Proc. IEEE 60, 1062 (1972).

${ }^{20}$ J. R. Dennis and E. B. Hale, J. Appl. Phys. 49, 1119 (1978).

${ }^{21}$ N. Hecking, K. F. Heidemann, and E. Te Kaat, Nucl. Instrum. Methods Phys. Res. B 15, 760 (1986).

${ }^{22}$ S. U. Campisano, S. Coffa, V. Raineri, F. Priolo, and E. Rimini, Nucl. Instrum. Methods Phys. Res. B 80/81, 514 (1993).

${ }^{23}$ E. Bezakova, A. P. Byrne, C. J. Glover, M. C. Ridgway, and R. Vianden, Appl. Phys. Lett. 75, 1923 (1999).

${ }^{24}$ K. Nordlund, M. Ghaly, R. S. Averback, M. Caturla, T. Diaz de la Rubia, and J. Tarus, Phys. Rev. B 57, 7556 (1998).

${ }^{25}$ K. Nordlund, J. Peltola, J. Nord, J. Keikonen, and R. S. Averback, J. Appl. Phys. 90, 1710 (2001).

${ }^{26}$ L. J. Lewis, A. De Vita, and R. Car, Phys. Rev. B 57, 1594 (1998). 